\title{
Complicações intra e pós-operatória da dacriocistorrinostomia externa
}

\author{
Complications intra and post operatory \\ of external dacryocystorhinostomy
}

Silvia Helena Tavares Lorena', João Amaro Ferrari Silva²

\section{RESUMO}

Objetivo: O objetivo do projeto de pesquisa é determinar a incidência das complicações intra e pós-operatória da dacriocistorrinostomia externa efetuadas pela autora, no Hospital São Paulo, no período de 10 de abril de 2009 a 15 de janeiro de 2010. Métodos:Estudo retrospectivo observacional dos prontuários dos pacientes submetidos à dacriocistorrinostomia externa no Hospital São Paulo, no período de 10 de abril de 2009 a 15 de janeiro de 2010. Os pacientes foram submetidos à avaliação oftalmológica: inspeção ocular, biomicroscopia,Teste de Milder, Teste de Jones I e II e exame de dacriocistografia. É mister salientar que os pacientes incluídos neste estudo foram examinados pela autora deste trabalho. Resultados: Neste trabalho, verificou-se que das 15 dacriocistorrinostomias externas realizadas as complicações intra-operatórias encontradas foram: sangramento(46,66\%), perda do retalho da mucosa nasal (26,66\%), utilização do martelo na osteotomia(6,66\%). Em relação as complicações pós-operatórias citamos: fístula cutânea $(6,66 \%)$, cicatriz hipertrófica $(6,66 \%)$. Observou-se 1 caso de falência, correspondendo a 6,66\% das cirurgias realizadas. O tempo médio de acompanhamento pós-operatório dos pacientes foi de 4 meses. Conclusão: Neste trabalho, o índice de sucesso obtido por esta técnica cirúrgica foi grande, podendo afirmar que a dacriocistorrinostomia externa oferece ótimos resultados.

Descritores: Dacriocistorrinostomia / efeitos adversos; Procedimentos cirúrgicos oftalmológicos/efeitos adversos; Obstrução dos ductos lacrimais/cirurgia; Complicações pós-operatórias

\footnotetext{
Abstract

Purpose: The research project goal is to determine the incidence of complications during the external dacryocystorhinostomy and post surgery done by the author, in São Paulo Hospital, from april $10^{\text {th }}, 2009$ to january 15 $5^{\text {th }}$,2010. Methods: This is a retrospective study of the records of the patients submitted to external dacryocystorhinostomy in São Paulo Hospital, from april $10^{\text {th }} 2009$ to january $15^{\text {th }}, 2010$. The patient were submitted to the ophtahalmologic evaluation: Ocular inspection, biomicroscopiy, Milder Test, Test of Jones I and II and dacryocystograph exam. It is important point out, that the included patients in this study, the patients were examined by the author of this work. Results: This study was verified that out of 15 external dacryocystorhinostomy done, the complications were: bleeding (46,66\%), oddment loss of the nasal mucosa $(26,66 \%$, utilização) of the hammer in the osteotomy $(6,66 \%)$. The pos-operatory complications: cutaneous fistula $(6,66 \%)$, hypertrophic $(6,66 \%)$. Among the 15 surgeries there was only 1 failure corresponding to the $6,66 \%$. The average time of surgery follow-up was of 4 months. Conclusion: The external dacryocystorhinostomy offers great results, according this study.

Keywords: Dacryocystorhinostomy/adverse effects; Ophthalmologic surgical procedures/adverse effects; Lacrimal duct obstruction/surgery; Postoperative complications

${ }^{1}$ Médica Colaboradora do Setor de Vias Lacrimais da Universidade Federal de São Paulo - (UNIFESP) - São Paulo (SP), Brasil; ${ }^{2}$ Chefe do Setor de Vias Lacrimais da Universidade Federal de São Paulo - (UNIFESP) - São Paulo (SP), Brasil.

Trabalho realizado no Setor de Vias Lacrimais da Universidade Federal de São Paulo - (UNIFESP) - São Paulo (SP), Brasil.

Os autores declaram inexistir conflitos de interesse

Recebido para publicação em 30/11/2010 - Aceito para publicação em 14/8/2011
}

Rev Bras Oftalmol. 2011; 70 (6): 404-7 


\section{INTRODUÇÃO}

A dacriocistorrinostomia externa é uma cirurgia destinada a efetuar a drenagem de lágrimas e de secreção do saco lacrimal no meato médio da fossa nasal através de uma osteotomia do osso da fossa lacrimal, realizando uma sutura dos retalhos da mucosa nasal com a do saco lacrimal.

Em 1904, foi realizada uma osteotomia da fossa lacrimal, com a extirpação das mucosas da parede do saco lacrimal e da mucosa nasal (dacriostomia) ${ }^{(1)}$. Em 1920, houve modificação da técnica acima descrita, realizando os retalhos da mucosa do saco lacrimal e da mucosa nasal e a sutura desses retalhos ${ }^{(2)}$. Em 1929 foi introduzida a técnica acima descrita no Brasil. Em 1967, foi introduzido o uso da entubação com silicone na dacriocistorrinostomia ${ }^{(3,4)}$.

Esta cirurgia é indicada na oclusão do ducto nasolacrimal em pessoas jovens e de meia idade, o qual está obstruído por tecido fibroso denso ou osso, justificando uma tentativa de estabelecer uma nova comunicação entre o saco lacrimal e a fossa nasal ${ }^{(5)}$.

A dacriocistorrinostomia externa pode ser realizada sob anestesia geral e internação hospitalar preferencialmente, ou sob anestesia local associada à sedação assistida pelo anestesista (lidocaína $2 \%$ com epinefrina 1:100.000 associado com bupivacaína $0,75 \%$ = solução anestésica de $5 \mathrm{ml}$, sendo $2 \mathrm{ml}$ no nervo infratroclear, $2 \mathrm{ml}$ no infraorbitário e $1 \mathrm{ml}$ na área da incisão.Usando um agulha mais longa (25x7), realizando infiltração mais profunda $(2,5 \mathrm{~cm})$, bloqueia o nervo nasociliar de onde emergem os nervos: infratroclear, etmoidal anterior e posterior. As etapas da cirurgia ${ }^{(6)}$ são:

- Incisão cutânea reta no dorso lateral do nariz, com extensão de 10-12 mm;

- Dissecção romba da musculatura orbicular;

- Abertura do periósteo, com bisturi de lâmina 11 a partir das extremidades da incisão;

- Dissecação do periósteo até a crista lacrimal anterior;

- Dissecção romba da musculatura orbicular;

- Abertura do periósteo, com bisturi de lâmina 11 a partir das extremidades da incisão;

- Dissecação da periósteo até a crista lacrimal anterior;

- Dissecação do saco lacrimal, desalojando-o de sua fossa até atingir a crista lacrimal posterior;

- Realização do retalho da mucosa do saco lacrimal;
- Osteotomia através da espátula de Donato Valle na linha de sutura entre o osso lacrimal e o processo frontal da maxila e introduzindo o osteótomo na abertura óssea;

- Realização do retalho da mucosa nasal;

- Sutura do retalho anterior do saco lacrimal com a margem do retalho nasal (vicryl 6.0);

- Sutura do plano muscular, com pontos separados e sepultados (vicryl 6.0);

- Sutura do plano cutâneo, com pontos contínuos (seda 7.0);

Atualmente, o tratamento da obstrução da via lacrimal excretora baixa é realizada principalmente pela técnica da dacriocistorrinostomia externa ${ }^{(7,8)}$.

A finalidade deste trabalho é determinar a incidência das complicações intra e pós-operatória da dacriocistorrinostomia externa efetuadas pela autora.

\section{Métodos}

Estudo retrospectivo observacional dos prontuários dos pacientes submetidos à dacriocistorrinostomia externa no Hospital São Paulo, no período de 10 de abril de 2009 a 15 de janeiro de 2010.Os pacientes foram submetidos à avaliação oftalmológica: inspeção ocular, biomicroscopia,Teste de Milder, Teste de Jones I e II e exame de dacriocistografia.

A inspeção ocular foi realizada com a finalidade de diagnosticar o mal posicionamento palpebral (entrópio, epibléfaro, ectrópio, paralisia facial) e na presença de massa, no canto medial ocular, verificar as suas características pela palpação (mole, dura, retrátil, pulsátil) e a presença de refluxo de secreção para a conjuntiva bulbar.

A biomicroscopia foi realizada, a fim de detectar uma pseudoepífora, que consiste num lacrimejamento reflexo, causado por: ceratite, triquíase, distiquíase, conjuntivite, corpo estranho e também observar a posição, estrutura, refluxo e coloração dos pontos lacrimais. Devemos salientar os seguintes testes:

- Teste do Desaparecimento da Fluoresceína(Teste de Milder)

Este teste consiste na instilação de uma gota de fluoresceína a $2 \%$ no fundo de saco conjuntival do olho suspeito, e o menisco lacrimal é examinado com um filtro de cobalto da lâmpada de fenda após cinco minutos. A retenção do corante varia de 0 a 4+ (meniscos de 0 a $1+$ são considerados normais; menisco de 2+ indica início de obstrução anatômica 


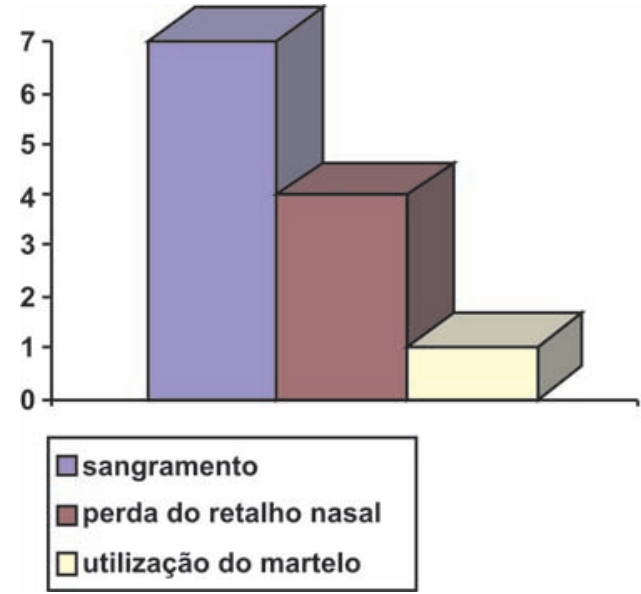

Figura 1: Complicações intraoperatórias da dacriocistorrinostomia externa

ou funcional; meniscos de $3+\mathrm{a} 4+$ indicam alteração do fluxo lacrimal).

-Teste de Jones I

Este teste avalia a permeabilidade da via lacrimal excretora e consiste na instilação de fluoresceína a $2 \%$ no fundo de saco conjuntival do olho suspeito de obstrução e após 5 minutos, iremos notar este corante na ponta de uma haste flexível que foi colocado no meato nasal inferior, previamente anestesiada a mucosa nasal com solução gel de xilocaína $2 \%$. Considera-se o teste positivo quando há o corante na ponta de uma haste flexível, indicando via lacrimal pérvia.

-Teste de Jones II

É realizado logo após o Teste de Jones I. O paciente deverá estar sentado, instila-se colírio anestésico tópico na conjuntiva bulbar, dilata-se o ponto lacrimal inferior do olho suspeito de obstrução e irriga-se a via lacrimal com $2 \mathrm{ml}$ de soro fisiológico através do canalículo inferior, observa-se o soro que sai pela fossa nasal. O soro colorido significa uma obstrução na via lacrimal excretora baixa.

A dacriocistografia constitui um importante exame para constatar radiologicamente a existência da obstrução, sendo empregado um contraste hidrossolúvel, iodado.

A primeira radiografia é realizada logo após a injeção do contraste, a segunda depois de 3 minutos, a terceira aos 5 minutos e a última aos 10 minutos.

A imagem do contraste é menos evidente na dacriocistografia aos 3 minutos e desaparece em $10 \mathrm{mi}-$ nutos nas vias lacrimais sem anormalidades anatômicas e funcionais.

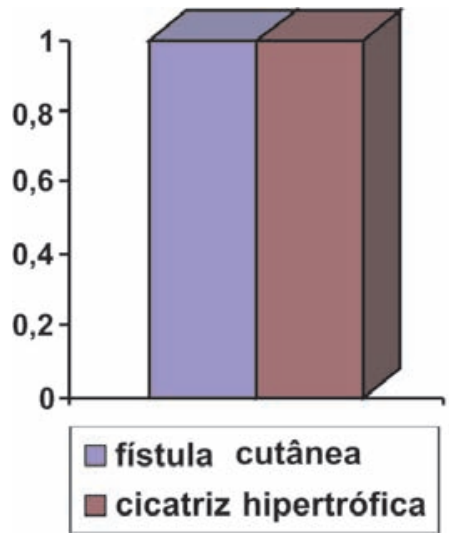

Figura 2: Complicações pós-operatórias da dacriocistorrinostomia externa

\section{Resultados}

Neste trabalho verificou-se que das 15 dacriocistorrinostomias externas realizadas as complicações intraoperatórias encontradas foram (Figura 1):

- sangramento(46,66\%),

- perda do retalho da mucosa nasal(26,66\%),

- utilização do martelo na osteotomia(6,66\%).

Em relação as complicações pós-operatórias citamos (Figura 2):

- fístula cutânea $(6,66 \%)$

- cicatriz hipertrófica no dorso lateral do na$\operatorname{riz}(6,66 \%)$.

Observou-se 1 caso de falência correspondendo a $6,66 \%$ das cirurgias realizadas.

O tempo médio de acompanhamento pós-operatório dos pacientes foi de 4 meses.

\section{Dıscussão}

Dos 15 pacientes submetidos à dacriocistorrinostomia externa, apenas 1 paciente apresentou antecedente de trauma em região medial da órbita, com bom resultado cirúrgico. Segundo a literatura ${ }^{(1)}$, os traumas possuem maior chance de insucesso devido às alterações anatômicas.

A literatura afirma índice de sucesso em dacriocistorrinostomia externa em pacientes com antecedente de trauma em torno de $33,0 \%{ }^{(2)}$ e na obstrução não traumática obteve-se em torno de $99,0 \%{ }^{(3)}$.

Em relação às complicações intraoperatórias, des- 
tacamos o sangramento ocorrido em $46,66 \%$ dos casos, dificultando a técnica cirúrgica e prolongando o tempo cirúrgico. O sangramento decorreu principalmente pela lesão da mucosa nasal provocada pelo osteótomo de Citelli no momento da osteotomia do osso nasal.

Os fatores que podem influenciar no índice de sucesso da dacriocistorrinostomia externa, além dos fatores intraoperatórios, salientamos: obstrução do canalículo comum, localização e tamanho da janela óssea, cicatrização da rinostomia, doença sistêmica ativa e outras $^{(3)}$.

No Setor de Vias Lacrimais da UNIFESP, não utilizamos a entubação nasolacrimal com sylastic como rotina nas cirurgias, contudo é utilizada somente nos casos de reoperação. Segundo a literatura, os índices de sucesso com a entubação nasolacrimal é de 91,6\% a $95,0 \%$ e de $2 \%$ nas reoperações ${ }^{(9)}$.

\section{Conclusões}

Neste trabalho, o índice de sucesso obtido por esta técnica cirúrgica foi grande, podendo afirmar que a dacriocistorrinostomia externa oferece ótimos resultados.

\section{REFERÊNCIAS}

1. Toti A. Nuovo metodo conservatore di cura radicale della supurazione croniche del sacco lacrimale (Dacriocistorrinostomia). Clín Méd. 1904;10:385-9.

2. Almeida A. Cirurgia do aparelho lacrimal. Arq Inst Penido Burnier. 1959;16:67-105.
3. Adenis JP, Mathon C, Lebraud P, Franco JL. [Dacryocystorhinostromy. Retrospective study of 165 cases. Indications. Technic. Results. Comparative study of 25 cases of injury with 165 cases including all etiologies]. J Fr Ophtalmol. 1987;10(4):323-9. French.

4. Adenis JP, Mathon C, Franco JL, Lebraudo P. Dacryocystorhinostomy for post-traumatic lacrimal stenosis: a study of 25 cases. Orbit. 1987;6(2):135-7.

5. Schellini SA, Itoda LK, Silva MRBM. Dacrioestenose: análise dos casos atendidos em nosso serviço. Rev Bras Oftalmol. 1990;49(6):43-8.

6. Pandya VB, Lee S, Benger R,Danks JJ, Kourt G,Martin PA, Lertsumitkul S, McCluskey P, Ghabrial R.External dacryocystorhinostomy: assessing factors that influence outcome. Orbit. 2010;29(5):291-7.

7. MacLachlan DL, Shannon GM, Flanagan JC. Results of dacryocystorhinostomy: analysis of the reoperations. Ophthalmic Surg. 1980;11(7):427-30.

8. Eisenbeis C, Neppert B, Menke T, Pape S, Scharader S, Geerling G, Muller M.Anatomic and subjective success of structured surgical treatment strategy in the management of chronic epiphora - a postoperative analysis of contentment. Klin Monbl Augenheilkd. 2010 Nov;227(11):879-86.

9. Gruss JJ, Hurwitz JJ, Nik NA, Kassel EE. The pattern and incidence of nasolacrimal injury in naso-orbital-ethmoid fractures: the role of delayed assessment and dacryocystorhinostomy. Br J Plast Surg. 1985;38(1):116-21.

Endereço para correspondência:

Silvia Helena Tavares Lorena

Rua Flórida, ${ }^{\circ} 1404$ - Brooklin

CEP 04561-030 - São Paulo (SP), Brasil.

Tel: (11) 5507-2705

E-mail: silvia.helenatavareslorena@yahoo.com.br 